\title{
Hemophilia Burden of Disease: A Systematic Review of the Cost-Utility Literature for Hemophilia
}

\author{
Teja Thorat, MSc, MPH; Peter J. Neumann, ScD; and James D. Chambers, PhD, MPharm, MSc
}

\begin{abstract}
BACKGROUND: Prophylaxis with clotting factor replacement products is recommended by the Medical and Scientific Advisory Council of the National Hemophilia Foundation as the optimal therapy for the prevention of bleeding episodes in individuals with severe hemophilia A or B $(<1$ IU per $\mathrm{dL}$ endogenous factor VIII or factor IX activity, respectively). Prophylaxis is associated with an improved health-related quality of life and has been shown to be cost-effective compared with on-demand therapy. However, the overall cost of treatment remains high, particularly among patients with a greater propensity to bleed. The overall value of hemophilia treatments and their associated benefits, measured in quality-adjusted life-years (QALYs), and dollar costs compared with other interventions can be evaluated through the use of cost-utility analyses (CUAs). Previous CUA studies in hemophilia have focused primarily on patients with more severe forms of hemophilia and on prophylaxis compared with on-demand treatment. However, to our knowledge, no studies to date have utilized QALYs as a standardized outcome measure to systematically evaluate the relative costeffectiveness of current hemophilia treatment options.
\end{abstract}

OBJECTIVE: To systematically review the CUA literature of hemophilia treatments and demonstrate the challenges in producing cost-utility evidence compared with other rare diseases.

METHODS: We conducted a systematic literature review using the Tufts Medical Center Cost-Effectiveness Analysis Registry and the National Health Service Economic Evaluation Database for English-language CUAs published from 2000 through 2015 with the search terms hemophilia, haemophilia, factor VIII, or factor IX. Two trained reviewers independently reviewed every study to extract relevant data. Incremental cost-effectiveness ratios were converted to 2014 U.S. dollars using exchange rates for currency conversion and the Consumer Price Index to adjust for inflation.

RESULTS: Our search yielded 52 studies, 11 of which met our inclusion criteria. The cost-effectiveness of hemophilia treatments varied widely based on variations in the study designs, including differences in time horizon, discount rates, and medical interventions.

CONCLUSIONS: We found the cost-effectiveness of hemophilia treatments to be broadly comparable to that of other orphan drugs. Improved standardization of future CUA studies will be important for further evaluation of the cost-effectiveness of hemophilia treatments.

J Manag Care Spec Pharm. 2018;24(7):632-42

Copyright $\odot 2018$, Academy of Managed Care Pharmacy. All rights reserved.

\section{What is already known about this subject}

Prophylactic therapy for hemophilia has been shown to be costeffective compared with on-demand therapy; however, the overall cost of treatment remains high, with the average cost of care for patients with hemophilia across different severity levels in the United States reported at almost $\$ 200,000$.

Because the vast majority of health care costs for patients with hemophilia can be attributed to factor replacement therapy, widespread adoption of prophylaxis replacement therapy in response to the 2007 Medical and Scientific Advisory Council of the National Hemophilia Foundation recommendations has contributed to increased medication costs to payers.

Previous cost-utility analyses examining hemophilia treatments have largely focused on patients with more severe forms of the disease and on comparing prophylaxis with on-demand treatment.

\section{What this study adds}

This study systematically reviewed the cost-utility analyses of hemophilia treatments, which showed that the cost utility of hemophilia treatments vary based on the treatment approach, patient characteristics, and disease severity.

The median incremental cost-effectiveness ratios reported across the literature varied by hemophilia type: $\$ 86,000$ per qualityadjusted life-years (QALY) for studies evaluating hemophilia A treatments, $\$ 17,000$ per QALY for a single study that evaluated treatment for hemophilia B, and $\$ 46,000$ per QALY for studies including patients with hemophilia A and B.

Identified estimates of the cost-effectiveness of hemophilia treatments are broadly comparable with those of other orphan drugs.

$\mathrm{O}$ rphan disease is a term referring to illnesses that affect only a small number of individuals within a geographic region (prevalence $\leq 50-85$ of every 100,000 individuals), although the specific numbers vary widely by country depending on their prevalence criteria for the definition of an orphan disease and their population size..$^{1-3}$ In the United States, orphan diseases are generally defined as those that affect fewer than 200,000 people. ${ }^{2,3}$ The number of orphan diseases worldwide is estimated to be between 5,000 and 8,000 , and only a small percentage of these have effective treatments available. $^{3}$ 
Hemophilia $\mathrm{A}$ is a rare, X-linked bleeding disorder that affects approximately 1 of every 5,000 to 10,000 live-born males. ${ }^{4}$ Hemophilia B is much less common than hemophilia A, with an incidence of approximately 1 in 25,000 births. $^{5}$ According to a 2016 global survey by the World Federation of Hemophilia, the total number of individuals with hemophilia worldwide was 184,723, including 16,949 within the United States. ${ }^{6}$ A definitive diagnosis of hemophilia A or B is typically made based on an established family history and/or patients' presentation of a bleeding event that has been confirmed by laboratory tests to be the result of coagulation factor deficiency. ${ }^{7}$ The clinical presentations of hemophilia A and B are indistinguishable, and the bleeding tendencies associated with each disorder tend to correlate directly with plasma concentrations of factor VIII (FVIII) and factor IX (FIX), respectively. Clotting factor levels of 5 to $40 \mathrm{IU}$ per dL confer mild hemophilia; factor levels of 1 to $5 \mathrm{IU}$ per $\mathrm{dL}$ are considered moderate, while factor levels $<1$ IU per dL define severe hemophilia. ${ }^{8,9}$ Severe hemophilia is characterized by spontaneous, recurrent bleeding into joints (hemarthrosis) and muscles. ${ }^{10}$ In subjects with severe hemophilia, hemarthrosis accounts for approximately $80 \%$ of all bleeding episodes., ${ }^{911,12}$ Over time, recurrent bleeding into the same joint leads to irreversible bone and cartilage damage, culminating in disabling hemophilic arthropathy. ${ }^{13,14}$

In addition, patients with severe hemophilia are at increased risk for developing high-affinity inhibitory antibodies (inhibitors) directed against FVIII or FIX, which neutralize the protective, coagulant effects of factor replacement therapy-the mainstay treatment for individuals with hemophilia. ${ }^{15}$ Overall, the prevalence of inhibitors among patients with mild and moderate hemophilia A or B is relatively low $(9 \%$ and $3 \%$, respectively); however, in patients with severe hemophilia, the incidence of inhibitors increases to as much as one-third in hemophilia A and 5\% in hemophilia B. ${ }^{15,16}$ The development of FIX inhibitors in hemophilia B gives rise to mild to severe allergic reaction during administration of FIX. ${ }^{15,16}$

Treatment for hemophilia A or B involves routine administration of exogenous coagulation factors to replace the missing/ deficient endogenous FVIII or FIX, respectively, ${ }^{4,7}$ The conventional treatment approach for hemophilia was on-demand (after the onset of bleeding) factor replacement; however, primary prophylaxis with replacement clotting factors has since become the standard of care for patients with severe hemophilia due to increasing evidence that this approach is associated with improved joint outcomes, decreased physical pain, and a better health-related quality of life (QOL) compared with on-demand therapy. ${ }^{17-19}$ In addition, prophylactic therapy has been shown to be cost-effective compared with on-demand therapy; however, the overall cost of treatment remains high, particularly among patients with more severe forms of hemophilia. ${ }^{20-22}$ A recent prospective, observational study $(\mathrm{N}=222)$ that examined the cost of care among people with mild to severe hemophilia A in hemophilia treatment centers in the United States found that the average annual direct costs for all individuals without inhibitors $(n=212)$ was $\$ 185,256$ per patient (in 2011 U.S. dollars [USD]). ${ }^{22}$ Ninety-two percent of the total direct medical costs and $80 \%$ of the overall costs were attributable to factor replacement therapy. The 2007 recommendation by the Medical and Scientific Advisory Council of the National Hemophilia Foundation that prophylaxis be considered optimal therapy for individuals with severe hemophilia A or B has also contributed to increased medication costs to payers due to the widespread adoption of prophylaxis replacement therapy. ${ }^{23,24}$ In a retrospective analysis of a U.S. health insurance claim database, the mean annual cost per patient in 2013 for individuals with hemophilia A and B was \$206,027 and \$179,747 (in 2012 USD), respectively. ${ }^{23}$ Approximately 25\%-30\% of patients with hemophilia A develop inhibitors to FVIII, while only 4\%-6\% of patients with hemophilia B develop inhibitors. ${ }^{22}$ The treatment cost for patients with inhibitors is even higher, with annual costs exceeding $\$ 400,000$ (in 2000 USD). ${ }^{25,26}$ Patients who develop inhibitors can be treated using immune tolerance therapy (ITT) or immune tolerance induction (ITI). ITI includes treating patients with frequent, sometimes extremely high, doses of repeated infusions of factor concentrates to eradicate inhibitors. ${ }^{4,27,28}$ The purpose of this form of treatment is to significantly reduce the presence of antibodies to the factor concentrates. ${ }^{27}$ Costs for ITI can exceed over $\$ 1$ million for patients with a good prognosis and can exceed more than $\$ 4$ million for patients with a poor prognosis (costs in 2000 USD). ${ }^{29}$

Pharmacoeconomic evaluations of treatment options in hemophilia can be performed in several different ways depending on the outcomes that are being measured. Cost-effectiveness analysis (CEA) is a technique used to measure the value of alternative health care interventions (e.g., primary prophylaxis vs. episodic treatment). ${ }^{30}$ CEAs examine costs and consequences of different medical interventions or programs relative to competing alternatives, helping decision makers prioritize certain interventions within a given budget. ${ }^{31}$ Alternatively, cost-utility analysis (CUA), a type of CEA, reports the value of health care interventions as incremental costs and incremental health benefits, measured in quality-adjusted life-years (QALYs), compared with alternative therapeutic approaches. The QALY is a standardized metric to measure health benefits, as it integrates QOL and survival gains. Previous cost-utility studies have focused on patients with severe hemophilia, prophylaxis compared with on-demand therapy, and specific treatments for targeted populations. ${ }^{21,32,33}$ This study systematically evaluated relative cost-effectiveness of hemophilia treatments using QALYs, a standardized health outcome measure.

The purpose of this study was to provide a systematic review of the CUA literature for hemophilia and demonstrate the challenges in producing cost-effectiveness evidence for hemophilia treatments compared with treatments for other rare diseases. 
PubMed and NHS EED database search for studies that evaluated hemophilia treatments, 1976-2015 $\mathrm{N}=52$

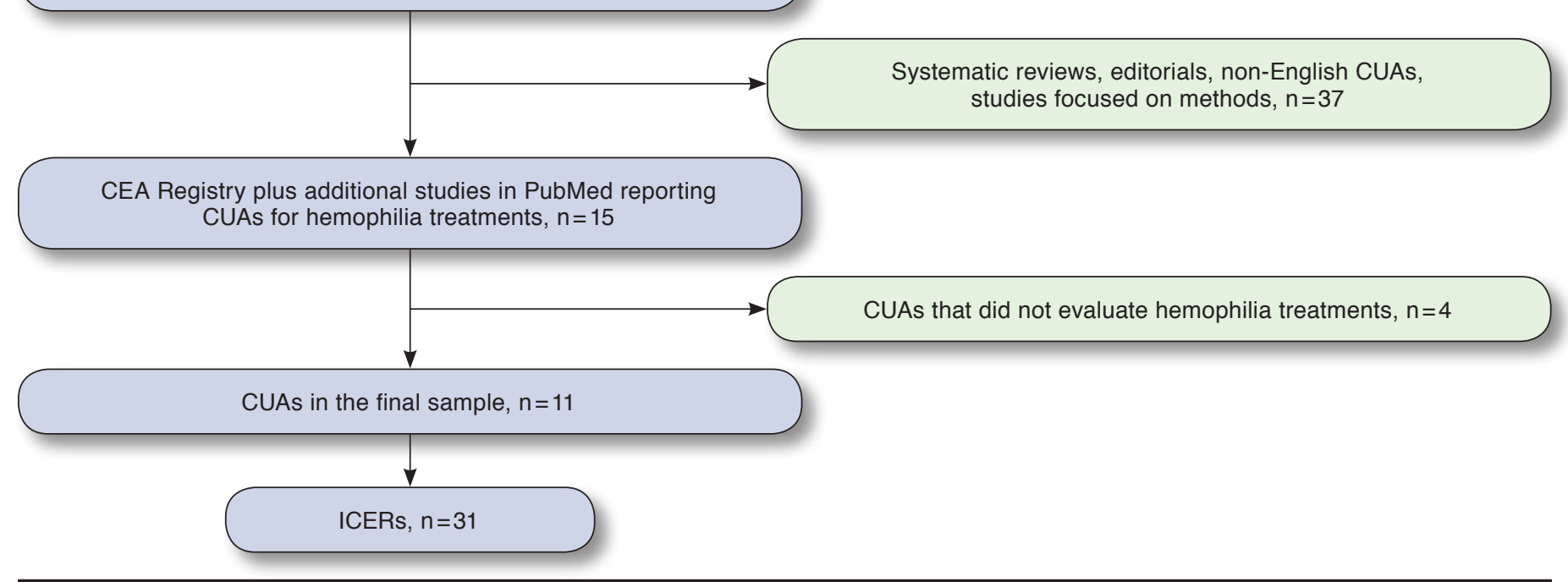

CEA = cost-effectiveness analysis; $C U A=$ cost-utility analysis; ICER = incremental cost-effectiveness ratio; NHS EED = National Health Services Economic Evaluation Database; QOL= quality of life.

\section{Methods}

\section{Search Strategy}

We conducted a systematic literature review using the Tufts Medical Center CEA Registry (www.cearegistry.org) and the National Health Service Economic Evaluation Database for English-language CUAs published from 2000 through 2015. The CEA Registry contains 5,000 English-language CUAs, with more than 13,400 standardized incremental cost-effectiveness ratios (ICERs) and 19,500 utility weights. The CEA Registry uses keywords such as QALYs, quality adjusted, and cost-utility analysis to search PubMed for English-language publications. Two trained reviewers independently reviewed every study to extract relevant data. Detailed information on the search strategy and data extraction for the CEA Registry is reported elsewhere. ${ }^{34,35}$ ICERs were converted to 2014 USD using exchange rates for currency conversion and the Consumer Price Index to adjust for inflation.

\section{Sample Selection and Data Analysis}

The search terms used to identify CUAs on hemophilia were hemophilia, haemophilia, factor VIII, or factor IX. Any reviews, meta-analyses, commentaries, or studies not reporting an original cost-per-QALY estimate for hemophilia treatment were excluded. CUAs were reviewed to summarize information on target population, intervention type, study sponsor, time horizon, study perspective, modeling approach, discount rate, and cost-effectiveness threshold. We calculated median ICERs by hemophilia type and treatment approach. The consort diagram describes the search strategy and sample selection process (Figure 1).

\section{Results}

Our search yielded 52 studies, 11 of which met our inclusion criteria (Table 1). Studies were most often set in the United Kingdom $(n=6)$, followed by Sweden $(n=2)$ and the United States $(n=2)$; other countries included were Italy, Iran, Thailand, and Germany. All CUAs evaluated pharmaceutical treatments. Eight studies estimated cost-effectiveness using a Markov model, incorporating health states that accounted for patients with and without inhibitors, patients requiring major surgery, and patients with major and minor bleeding events. Markov models are used in decision analysis, commonly for economic evaluations of medical interventions. They consist of different health states representing the consequences of the intervention in the disease under consideration. Cohorts of patients transition between the disease states depending on the transition probabilities and the cycle length stated in the model. They are useful for modeling chronic diseases. Five studies used 1-year Markov cycles, and 3 studies used 3-month Markov cycles.

The majority of CUAs discounted both costs (73\%) and QALYs (64\%). The most common discount rates used were 3\% 


\section{TABLE 1 CUA Studies in Hemophilia by Methodological Approach}

\begin{tabular}{|c|c|c|c|c|c|c|c|c|c|}
\hline Study & Country & Funder & $\begin{array}{l}\text { Perspective } \\
\text { (Resource Use/ } \\
\text { Costs) }\end{array}$ & $\begin{array}{l}\text { Study } \\
\text { Design }\end{array}$ & $\begin{array}{c}\text { Time } \\
\text { Horizon }\end{array}$ & Discounting & $\begin{array}{c}\text { CE } \\
\text { Threshold }\end{array}$ & $\begin{array}{l}\text { Health States for } \\
\text { Markov Model }\end{array}$ & $\begin{array}{l}\text { Cycle } \\
\text { Length }\end{array}$ \\
\hline $\begin{array}{l}\text { Ekert et al. } \\
(2001)^{51}\end{array}$ & Australia & $\begin{array}{l}\text { Pharmaceutical } \\
\text { company, Novo } \\
\text { Nordisk }\end{array}$ & $\begin{array}{l}\text { Health care payer } \\
\text { (medication, other } \\
\text { health care services } \\
\text { [e.g., pediatrician } \\
\text { consultations, } \\
\text { emergency room } \\
\text { visits, inpatient } \\
\text { stays]) }\end{array}$ & \begin{tabular}{|c|}
$\begin{array}{c}\text { Longitudinal } \\
\text { study }\end{array}$ \\
\\
\end{tabular} & \begin{tabular}{|c|}
18 \\
months
\end{tabular} & $\begin{array}{l}\text { Costs: none } \\
\text { QALYs: none }\end{array}$ & Not stated & Not applicable & $\begin{array}{c}\text { Not } \\
\text { applicable }\end{array}$ \\
\hline $\begin{array}{l}\text { Miners et al. } \\
(2002)^{38}\end{array}$ & $\begin{array}{l}\text { United } \\
\text { Kingdom }\end{array}$ & Not stated & $\begin{array}{l}\text { Societal (surgery, } \\
\text { medications [clot- } \\
\text { ting factors], } \\
\text { outpatient visits, } \\
\text { inpatient visits, } \\
\text { day-case visits, } \\
\text { productivity losses) }\end{array}$ & $\begin{array}{l}\text { Markov } \\
\text { model }\end{array}$ & Lifetime & $\begin{array}{l}\text { Costs: 6\% } \\
\text { QALYs: 6\% }\end{array}$ & Not stated & $\begin{array}{l}\text { Alive, requiring major } \\
\text { surgery, surgery, dead }\end{array}$ & 1 year \\
\hline $\begin{array}{l}\text { Knight et al. } \\
(2003)^{52}\end{array}$ & $\begin{array}{l}\text { United } \\
\text { Kingdom }\end{array}$ & $\begin{array}{l}\text { Government, } \\
\text { DHSC, } \\
\text { London, } \\
\text { Research and } \\
\text { Development }\end{array}$ & $\begin{array}{l}\text { Health care payer } \\
\text { (hemostatic agents, } \\
\text { inpatient stay, } \\
\text { arthropathy treat- } \\
\text { ment) }\end{array}$ & $\begin{array}{l}\text { Markov } \\
\text { model }\end{array}$ & Lifetime & $\begin{array}{c}\text { Costs: } 6 \% \\
\text { QALYs: } 1.5 \%\end{array}$ & $£ 30,000$ & $\begin{array}{l}\text { Hemophilia A with } \\
\text { inhibitor level BU } \geq 10 \\
\text { (high responders), hemo- } \\
\text { philia A with inhibi- } \\
\text { tor level BU }<10 \text { (low } \\
\text { responders), hemophilia } \\
\text { A without inhibitors. The } \\
\text { complications associated } \\
\text { with each health state } \\
\text { are minor bleeds, major } \\
\text { bleeds, arthropathy, } \\
\text { death }\end{array}$ & 3 months \\
\hline $\begin{array}{l}\text { Lippert et al. } \\
(2005)^{32}\end{array}$ & \begin{tabular}{|l|} 
Germany, \\
the \\
Netherlands, \\
Sweden, \\
United \\
Kingdom \\
\end{tabular} & $\begin{array}{l}\text { Pharmaceutical } \\
\text { company, } \\
\text { Aventis Behring }\end{array}$ & $\begin{array}{l}\text { Health care payer } \\
\text { (medication [anti- } \\
\text { coagulation factor], } \\
\text { hospitalization, } \\
\text { outpatient treat- } \\
\text { ment) }\end{array}$ & $\begin{array}{l}\text { Decision- } \\
\text { tree model }\end{array}$ & 1 year & $\begin{array}{l}\text { Costs: NA } \\
\text { QALYs: NA }\end{array}$ & Not stated & Not stated & $\begin{array}{c}\text { Not } \\
\text { applicable }\end{array}$ \\
\hline $\begin{array}{l}\text { Risebrough } \\
\text { et al. }(2008)^{33}\end{array}$ & Canada & $\begin{array}{l}\text { Pharmaceutical } \\
\text { company, Bayer }\end{array}$ & $\begin{array}{l}\text { Societal (FVIII, } \\
\text { professional visits } \\
\text { and tests, central } \\
\text { venous placement/ } \\
\text { complications, hos- } \\
\text { pitalization, home } \\
\text { programs, parents' } \\
\text { lost work days) }\end{array}$ & $\begin{array}{l}\text { Markov } \\
\text { model }\end{array}$ & 5 years & $\begin{array}{l}\text { Costs: 3\% } \\
\text { QALYs: 3\% }\end{array}$ & Not stated & $\begin{array}{l}\text { Development of up to } 3 \\
\text { target joints ( } 3 \text { bleeds in } \\
\text { a joint over } 3 \text { months) }\end{array}$ & 3 months \\
\hline $\begin{array}{l}\text { Miners et al. } \\
(2009)^{39}\end{array}$ & $\begin{array}{l}\text { United } \\
\text { Kingdom }\end{array}$ & $\begin{array}{l}\text { Pharmaceutical } \\
\text { company, } \\
\text { Baxter } \\
\text { Healthcare }\end{array}$ & $\begin{array}{l}\text { Health care payer } \\
\text { (clotting factor, } \\
\text { outpatient and } \\
\text { inpatient stays) }\end{array}$ & $\begin{array}{l}\text { Markov } \\
\text { model }\end{array}$ & $\begin{array}{l}\text { Lifetime } \\
(70 \\
\text { years })\end{array}$ & $\begin{array}{l}\text { Costs: } 3.5 \% \\
\text { QALYs: } 3.5 \%\end{array}$ & $\begin{array}{l}£ 30,000 \\
£ 40,000 \\
£ 100,000\end{array}$ & $\begin{array}{l}\text { Alive, year prior to major } \\
\text { surgery, year in which } \\
\text { major surgery is under- } \\
\text { taken, dead (same model } \\
\text { as Miners et al., 2002 }\end{array}$ & 1 year \\
\hline $\begin{array}{l}\text { Rasekh et al. } \\
(2011)^{53}\end{array}$ & Iran & $\begin{array}{l}\text { University, } \\
\text { Shahid } \\
\text { Beheshti } \\
\text { University } \\
\text { of Medical } \\
\text { Sciences }\end{array}$ & $\begin{array}{l}\text { Health care payer } \\
\text { (clotting factors) }\end{array}$ & $\begin{array}{l}\text { Markov } \\
\text { model } \\
\text { (based on } \\
\text { Knight } \\
\text { et al. }{ }^{52} \text { ) }\end{array}$ & 10 years & $\begin{array}{l}\text { Costs: none } \\
\text { QALYs: none }\end{array}$ & Not stated & $\begin{array}{l}\text { Hemophilia A with } \\
\text { inhibitor level BU } \geq 10 \\
\text { (high responders), hemo- } \\
\text { philia A with inhibi- } \\
\text { tor level BU }<10 \text { (low } \\
\text { responders), hemophilia } \\
\text { A without inhibitors. The } \\
\text { complications associated } \\
\text { with each health state } \\
\text { are minor bleeds, major } \\
\text { bleeds, arthropathy, } \\
\text { death }\end{array}$ & 3 months \\
\hline
\end{tabular}

continued on next page 
Hemophilia Burden of Disease: A Systematic Review of the Cost-Utility Literature for Hemophilia

TABLE 1 CUA Studies in Hemophilia by Methodological Approach (continued)

\begin{tabular}{|c|c|c|c|c|c|c|c|c|c|}
\hline Study & Country & Funder & $\begin{array}{c}\text { Perspective } \\
\text { (Resource Use/ } \\
\text { Costs) }\end{array}$ & $\begin{array}{l}\text { Study } \\
\text { Design }\end{array}$ & $\begin{array}{c}\text { Time } \\
\text { Horizon }\end{array}$ & Discounting & $\begin{array}{c}\text { CE } \\
\text { Threshold }\end{array}$ & $\begin{array}{l}\text { Health States for } \\
\text { Markov Model }\end{array}$ & $\begin{array}{l}\text { Cycle } \\
\text { Length }\end{array}$ \\
\hline $\begin{array}{l}\text { Colombo et al. } \\
(2011)^{20}\end{array}$ & Italy & $\begin{array}{l}\text { Pharmaceutical } \\
\text { company, } \\
\text { Pfizer }\end{array}$ & $\begin{array}{l}\text { Health care payer } \\
\text { (recombinant } \\
\text { plasma/albumin- } \\
\text { free, hospitaliza- } \\
\text { tions for bleedings } \\
\text { or examinations, } \\
\text { hospitalizations for } \\
\text { major surgery) }\end{array}$ & $\begin{array}{l}\text { Markov } \\
\text { model }\end{array}$ & \begin{tabular}{|} 
Lifetime \\
$(70$ \\
years $)$
\end{tabular} & $\begin{array}{l}\text { Costs: } 6 \% \\
\text { QALYs: not } \\
\text { stated }\end{array}$ & $\begin{array}{l}€ 36,500 \\
€ 60,000 \\
€ 25,000- \\
€ 40,000\end{array}$ & $\begin{array}{l}\text { Alive, requiring major } \\
\text { surgery, surgery, dead }\end{array}$ & 1 year \\
\hline $\begin{array}{l}\text { Farrugia et al. } \\
(2013)^{21}\end{array}$ & $\begin{array}{l}\text { United } \\
\text { States, } \\
\text { United } \\
\text { Kingdom, } \\
\text { Sweden }\end{array}$ & $\begin{array}{l}\text { Pharmaceutical } \\
\text { company (mul- } \\
\text { tiple sources, } \\
\text { unclear) }\end{array}$ & $\begin{array}{l}\text { Health care payer } \\
\text { (FVIII, prothrom- } \\
\text { bin complex con- } \\
\text { centrate, orthope- } \\
\text { dic surgery) }\end{array}$ & $\begin{array}{l}\text { Markov } \\
\text { model }\end{array}$ & $\begin{array}{l}\text { Lifetime } \\
\text { (100 } \\
\text { years) }\end{array}$ & $\begin{array}{l}\text { Costs: } 3 \% \\
\text { (U.S. and } \\
\text { Sweden), 3.5\% } \\
\text { (U.K.) } \\
\text { QALYs: 3\% } \\
\text { (U.S. and } \\
\text { Sweden), 1.5\% } \\
\text { (U.K.) }\end{array}$ & $\begin{array}{l}£ 30,000 \\
\$ 50,000- \\
\$ 200,000\end{array}$ & $\begin{array}{l}\text { Alive, no inhibitors; alive } \\
\text { with inhibitors; dead. } \\
\text { Also, on-demand arm } \\
\text { includes orthopedic sur- } \\
\text { gery and major bleeding } \\
\text { events }\end{array}$ & 1 year \\
\hline $\begin{array}{l}\text { Pattanaprateep } \\
\text { et al. }(2014)^{54}\end{array}$ & Thailand & $\begin{array}{l}\text { Foundation, } \\
\text { HITAP }\end{array}$ & $\begin{array}{l}\text { Health care payer } \\
\text { (factor concentrate } \\
\text { costs at home, } \\
\text { emergency surgery; } \\
\text { life-threatening } \\
\text { operations; and } \\
\text { other hospital } \\
\text { treatments, such as } \\
\text { blood components; } \\
\text { medical supplies; } \\
\text { costs for laboratory } \\
\text { tests, room, doc- } \\
\text { tor fee) }\end{array}$ & $\begin{array}{l}\text { Markov } \\
\text { model }\end{array}$ & Lifetime & $\begin{array}{l}\text { Costs: 3\% } \\
\text { QALYs: 3\% }\end{array}$ & $\begin{array}{l}\text { Thai baht } \\
120,000\end{array}$ & $\begin{array}{l}\text { Well, life-threatening } \\
\text { bleeding (hemorrhage } \\
\text { or bleeding leading } \\
\text { to death, or requiring } \\
\text { prompt hospital inter- } \\
\text { vention), emergency } \\
\text { surgery (surgical proce- } \\
\text { dure that risks bleeding), } \\
\text { death }\end{array}$ & 1 year \\
\hline $\begin{array}{l}\text { Earnshaw } \\
\text { et al. }(2015)^{55}\end{array}$ & $\begin{array}{l}\text { United } \\
\text { States }\end{array}$ & $\begin{array}{l}\text { Pharmaceutical } \\
\text { company, } \\
\text { Grifols }\end{array}$ & $\begin{array}{l}\text { Health care payer } \\
\text { (drug costs, factor } \\
\text { inhibitor test, hospi- } \\
\text { talization, arthopa- } \\
\text { thy surgery, inhibi- } \\
\text { tor monitoring) }\end{array}$ & Decision tree & Lifetime & $\begin{array}{l}\text { Costs: } 3 \% \\
\text { QALYs: } 3 \%\end{array}$ & $\begin{array}{l}\$ 50,000 \\
\$ 100,000\end{array}$ & $\begin{array}{l}\text { Inhibitors < } 10 \mathrm{BU}, \\
\text { inhibitors > } 10 \mathrm{BU}, \\
\text { successful primary ITI, } \\
\text { primary ITI, successful } \\
\text { secondary ITI, failure of } \\
\text { secondary ITI }\end{array}$ & $\begin{array}{c}\text { Not } \\
\text { applicable }\end{array}$ \\
\hline
\end{tabular}

$B U=$ Bethesda unit; $C E=$ cost-effectiveness; $C U A=$ cost-utility analysis; DHSC = Department of Health and Social Care; FVIII = factor VIII; HITAP = Health Intervention and Technology Assessment Program; ITI = immune tolerance induction; NA=not applicable; QALY=quality-adjusted life-year; U.K. =United Kingdom; U.S. =United States.

$(\mathrm{n}=4)$ and $6 \%(\mathrm{n}=3)$ for costs and 3\% $(\mathrm{n}=4)$ for QALYs. A lifetime time horizon was most commonly applied (7 CUAs). Nine CUAs were performed using a health care perspective (direct medical costs included those for clotting factors, hospitalizations, tests, consultations), and 2 CUAs were performed using a societal perspective (productivity losses were additionally accounted for). Six CUAs compared estimated ICERs with a costeffectiveness threshold; the most commonly used threshold was $£ 30,000$ (approximately $\$ 50,000$ ), which was used in 3 studies.

\section{Estimated Cost-Effectiveness}

Eight CUAs evaluated hemophilia A treatments, 1 CUA evaluated hemophilia B treatment, and 3 did not differentiate between hemophilia A and B (Table 2). In studies that did not differentiate between patients with hemophilia A and B, patients were predominantly suffering from hemophilia A. Eight CUAs included treatments for patients with severe hemo- philia A, and 4 studies included patients with inhibitors. The studied target populations included male patients at birth or aged $\leq 2$ years, as well as adolescents (aged 11-17 years) and adolescents/adults (aged $\geq 14$ years).

Among the available therapeutic approaches for hemophilia, the CUAs studied prophylactic treatment or on-demand therapy with clotting factors, such as FVIII, recombinant FVIII (rFVIII), or factor VIIa (FVIIa) for hemophilia A; activated prothrombin complex concentrates (APCC); and ITI with rFVIII to eradicate inhibitors plus FVIII to control bleeds and FIX for hemophilia B. All studies compared prophylactic treatment or ITI to on-demand therapy. There was large variation in the interventions reported in the CUAs, including different protocols for ITI, different frequencies of prophylaxis (daily and every 2 days), or combination of on-demand and prophylaxis with changes in the treatment pattern. 


\section{TABLE 2 CUA Studies in Hemophilia by OALY and ICER Outcome Measures}

\begin{tabular}{|c|c|c|c|c|c|c|c|}
\hline \multirow[b]{2}{*}{ Study } & \multicolumn{2}{|c|}{ Patient Population } & \multirow[b]{2}{*}{ Intervention vs. Comparator } & \multirow[b]{2}{*}{$\begin{array}{c}\text { Country, Currency } \\
\text { Year }\end{array}$} & \multirow{2}{*}{$\begin{array}{l}\text { Incremental } \\
\text { Costs in Year } \\
\text { and Currency } \\
\text { Stated in Study }\end{array}$} & \multirow{2}{*}{$\begin{array}{l}\text { Incremental } \\
\text { QALYs }\end{array}$} & \multirow{2}{*}{$\begin{array}{l}\text { ICER in Year and } \\
\text { Currency Stated } \\
\text { in Study } \\
\text { (USD 2014) }\end{array}$} \\
\hline & $\begin{array}{l}\text { Hemophilia } \\
\text { Type }\end{array}$ & $\begin{array}{l}\text { Age and Other } \\
\text { Comorbidities }\end{array}$ & & & & & \\
\hline \multicolumn{8}{|c|}{ Hemophilia A } \\
\hline $\begin{array}{l}\text { Miners et al. } \\
(2002)^{38}\end{array}$ & $\begin{array}{l}\text { Severe } \\
\text { hemophilia A/ } \\
\text { severe von } \\
\text { Willebrand's } \\
\text { disease }\end{array}$ & At birth & $\begin{array}{l}\text { Prophylaxis vs. on-demand } \\
\text { management }\end{array}$ & $\begin{array}{l}\text { United Kingdom, } \\
2000 \text { (pound) }\end{array}$ & 694,070 & 3.340 & $\begin{array}{c}46,500 \\
(96,000)^{\mathrm{a}}\end{array}$ \\
\hline \multirow{6}{*}{$\begin{array}{l}\text { Knight et al. } \\
(2003)^{52}\end{array}$} & \multirow{6}{*}{$\begin{array}{l}\text { Severe } \\
\text { hemophilia A } \\
\text { with high- } \\
\text { responding } \\
\text { inhibitors }\end{array}$} & \multirow{6}{*}{2 years } & $\begin{array}{l}\text { Bonn (high-dose) ITI protocolb vs. } \\
\text { on-demand management with APCC } \\
\text { and pFVIII }\end{array}$ & \multirow{6}{*}{$\begin{array}{l}\text { United Kingdom, } \\
2000 \text { (pound) }\end{array}$} & $1,177,144$ & 7.900 & $\begin{array}{l}147,785 \\
(308,000)\end{array}$ \\
\hline & & & $\begin{array}{l}\text { Low-dose ITI protocolc vs. on- } \\
\text { demand management with APCC } \\
\text { and pFVIII }\end{array}$ & & 223,949 & 4.000 & $\begin{array}{c}55,922 \\
(116,000)\end{array}$ \\
\hline & & & $\begin{array}{l}\text { Malmo ITI protocold vs. on-demand } \\
\text { management with APCC and pFVIII }\end{array}$ & & $-137,708$ & 3.000 & Dominant \\
\hline & & & $\begin{array}{l}\text { Bonn (high-dose) ITI protocolb vs. } \\
\text { on-demand management with rFVIIa }\end{array}$ & & $1,360,408$ & 7.900 & $\begin{array}{c}170,793 \\
(355,000)\end{array}$ \\
\hline & & & $\begin{array}{l}\text { Low-dose ITI protocolc vs. on- } \\
\text { demand management with rFVIIa }\end{array}$ & & 327,306 & 4.000 & $\begin{array}{c}81,731 \\
(169,000) \\
\end{array}$ \\
\hline & & & $\begin{array}{l}\text { Malmo ITI protocold vs. on-demand } \\
\text { management with rFVIIa }\end{array}$ & & $-60,453$ & 3.000 & Dominant \\
\hline \multirow[b]{2}{*}{$\begin{array}{l}\text { Risebrough } \\
\text { et al. }(2008)^{33}\end{array}$} & \multirow{2}{*}{$\begin{array}{l}\text { Severe } \\
\text { hemophilia A } \\
\text { (FVIII <2 } \\
\text { IU/dL) }\end{array}$} & \multirow[b]{2}{*}{1 year } & $\begin{array}{l}\text { Tailored prophylaxis (escalating dose)e } \\
\text { vs. on-demand therapy ( } 40 \mathrm{IU} / \mathrm{kg} \\
\text { upon presentation of bleeding and } 20 \\
\text { IU/kg on Days } 1 \text { and } 3 \text { postbleed) }\end{array}$ & \multirow{2}{*}{$\mid \begin{array}{c}\text { Canada, 2003 } \\
\text { (Canadian dollars) }\end{array}$} & 165,976 & 0.300 & $\begin{array}{l}542,938 \\
(500,000)\end{array}$ \\
\hline & & & $\begin{array}{l}\text { Standard prophylaxis (FVIII } 25 \text { IU/kg } \\
\text { thrice weekly) vs. on-demand ther- } \\
\text { apy ( } 40 \text { IU/kg upon presentation of } \\
\text { bleeding and } 20 \text { IU/kg on Days } 1 \\
\text { and } 3 \text { postbleed) }\end{array}$ & & 292,626 & 0.310 & $\begin{array}{c}943,954 \\
(869,000)\end{array}$ \\
\hline $\begin{array}{l}\text { Miners et al. } \\
(2009)^{39}\end{array}$ & $\begin{array}{l}\text { Severe } \\
\text { hemophilia A }\end{array}$ & At birth & $\begin{array}{l}\text { Prophylactic treatment vs. on- } \\
\text { demand treatment with FVIII }\end{array}$ & $\begin{array}{l}\text { United Kingdom, } \\
2007 \text { (pound) }\end{array}$ & 214,000 & 5.630 & $\begin{array}{l}38,000 \\
(86,000)\end{array}$ \\
\hline \multirow{3}{*}{$\begin{array}{l}\text { Rasekh et al. } \\
(2011)^{53}\end{array}$} & \multirow{3}{*}{$\begin{array}{l}\text { Severe } \\
\text { hemophilia A } \\
\text { with high- } \\
\text { titer } \\
\text { inhibitors }\end{array}$} & \multirow{3}{*}{2 years } & $\begin{array}{l}\text { Bonn (high-dose) ITI protocolb vs. } \\
\text { on-demand treatment with rFVIIa }\end{array}$ & \multirow{3}{*}{$\begin{array}{l}\text { Iran, } 2011 \\
\text { (U.S. dollars) }\end{array}$} & $-676,598$ & 7.900 & Dominant \\
\hline & & & $\begin{array}{l}\text { Low-dose ITI protocolf vs. } \\
\text { on-demand treatment with rFVIIa }\end{array}$ & & $-3,961,598$ & 4.000 & Dominant \\
\hline & & & $\begin{array}{l}\text { Malmo ITI protocold vs. on-demand } \\
\text { treatment with rFVIIa }\end{array}$ & & $-1,898,618$ & 3.000 & Dominant \\
\hline \multirow{3}{*}{$\begin{array}{l}\text { Colombo et al. } \\
(2011)^{20}\end{array}$} & \multirow{3}{*}{$\begin{array}{l}\text { Severe } \\
\text { hemophilia A }\end{array}$} & \multirow{3}{*}{ At birth } & $\begin{array}{l}\text { Primary prophylaxis with FVIII con- } \\
\text { centrates vs. on-demand treatment } \\
\text { with FVIII infusions }\end{array}$ & \multirow{3}{*}{$\begin{array}{l}\text { Italy, } 2010 \\
\quad \text { (euro) }\end{array}$} & 787,420 & 19.570 & $\begin{array}{l}40,236 \\
(58,000)\end{array}$ \\
\hline & & & $\begin{array}{l}\text { Secondary prophylaxis with FVIII } \\
\text { concentrates vs. on-demand treat- } \\
\text { ment with FVIII infusions }\end{array}$ & & 770,140 & 19.140 & $\begin{array}{l}40,229 \\
(58,000)\end{array}$ \\
\hline & & & $\begin{array}{l}\text { Hybrid regimen with FVIII concen- } \\
\text { trates vs. on-demand treatment with } \\
\text { FVIII infusions }\end{array}$ & & 421,734 & 3.540 & $\begin{array}{l}119,134 \\
(170,000)\end{array}$ \\
\hline \multirow{4}{*}{$\begin{array}{l}\text { Farrugia et al. } \\
(2013)^{21}\end{array}$} & \multirow{4}{*}{$\begin{array}{l}\text { Severe } \\
\text { hemophilia A }\end{array}$} & \multirow{4}{*}{ At birth } & \multirow{3}{*}{$\begin{array}{l}\text { Prophylaxis every } 2 \text { days vs. } \\
\text { on-demand therapy }\end{array}$} & $\begin{array}{c}\text { United States, } 2011 \\
\text { (U.S. dollars) }\end{array}$ & 412,999 & 6.060 & $\begin{array}{c}68,109 \\
(71,000)\end{array}$ \\
\hline & & & & \begin{tabular}{|c|}
$\begin{array}{c}\text { United Kingdom, } \\
2011 \text { (pound) }\end{array}$ \\
\end{tabular} & $-280,866$ & 9.690 & Dominant \\
\hline & & & & $\begin{array}{l}\text { Sweden, } 2011 \\
\text { (Swedish krona) }\end{array}$ & $5,331,051$ & 10.990 & $\begin{array}{l}484,888 \\
(78,000) \\
\end{array}$ \\
\hline & & & $\begin{array}{l}\text { Daily prophylaxis dosing vs. } \\
\text { on-demand therapy }\end{array}$ & $\begin{array}{l}\text { Sweden, } 2011 \\
\text { (Swedish krona) }\end{array}$ & $-10,541,993$ & 10.990 & Dominant \\
\hline \multirow{2}{*}{$\begin{array}{l}\text { Earnshaw et } \\
\text { al. }(2015)^{55}\end{array}$} & \multirow{2}{*}{$\begin{array}{l}\text { Severe } \\
\text { hemophilia A } \\
\text { with } \\
\text { inhibitors }\end{array}$} & \multirow{2}{*}{ Infant } & $\begin{array}{l}\text { Prophylaxis every } 2 \text { days vs. } \\
\text { on-demand therapy }\end{array}$ & \multirow{2}{*}{$\begin{array}{c}\text { United States, } 2014 \\
\text { (U.S. dollars) }\end{array}$} & $-1,637,240$ & 9.9 & Dominant \\
\hline & & & $\begin{array}{l}\text { Daily prophylaxis dosing vs. } \\
\text { on-demand therapy }\end{array}$ & & $-23,201,543$ & 4.3 & Dominant \\
\hline
\end{tabular}


TABLE 2 CUA Studies in Hemophilia by QALY and ICER Outcome Measures (continued)

\begin{tabular}{|c|c|c|c|c|c|c|c|}
\hline \multirow[b]{2}{*}{ Study } & \multicolumn{2}{|c|}{ Patient Population } & \multirow[b]{2}{*}{ Intervention vs. Comparator } & \multirow[b]{2}{*}{$\begin{array}{l}\text { Country, Currency } \\
\text { Year }\end{array}$} & \multirow{2}{*}{$\begin{array}{c}\text { Incremental } \\
\text { Costs in Year } \\
\text { and Currency } \\
\text { Stated in Study }\end{array}$} & \multirow[b]{2}{*}{$\begin{array}{c}\text { Incremental } \\
\text { QALYs }\end{array}$} & \multirow{2}{*}{$\begin{array}{l}\text { ICER in Year and } \\
\text { Currency Stated } \\
\text { in Study } \\
\text { (USD 2014) }\end{array}$} \\
\hline & $\begin{array}{l}\text { Hemophilia } \\
\text { Type }\end{array}$ & $\begin{array}{l}\text { Age and Other } \\
\text { Comorbidities }\end{array}$ & & & & & \\
\hline \multicolumn{8}{|l|}{ Hemophilia B } \\
\hline \begin{tabular}{l|} 
Miners et al. \\
$(2002)^{38}$
\end{tabular} & $\begin{array}{l}\text { Severe } \\
\text { hemophilia B }\end{array}$ & At birth & $\begin{array}{l}\text { Prophylaxis with clotting factors vs. } \\
\text { on-demand with clotting factors }\end{array}$ & $\begin{array}{l}\text { United Kingdom, } \\
2000 \text { (pound) }\end{array}$ & 133,818 & 3.340 & $\begin{array}{c}8,600 \\
(17,000)^{\mathrm{a}}\end{array}$ \\
\hline \multicolumn{8}{|c|}{ Hemophilia A and B } \\
\hline $\begin{array}{l}\text { Ekert et al. } \\
(2001)^{51}\end{array}$ & $\begin{array}{l}\text { Hemophilia A } \\
(\mathrm{n}=5) \text { and } \mathrm{B} \\
(\mathrm{n}=1) \text { with } \\
\text { long-standing } \\
\text { inhibitors }\end{array}$ & $11-17$ years & $\begin{array}{l}\text { Prophylaxis with rFVIIa vs. } \\
\text { on-demand usual care }\end{array}$ & $\begin{array}{c}\text { Australia, } 1999 \\
\text { (Australian dollars) }\end{array}$ & 29,901 & 0.580 & $\begin{array}{c}51,553 \\
(46,000)\end{array}$ \\
\hline \multirow{4}{*}{$\begin{array}{l}\text { Lippert et al. } \\
(2005)^{32}\end{array}$} & \multirow{4}{*}{$\begin{array}{l}\text { Severe } \\
\text { hemophilia A } \\
(84 \%) \text { and B } \\
(16 \%) \text {, with- } \\
\text { out inhibitors }\end{array}$} & $\begin{array}{l}\leq 30 \text { years, } \\
\text { HIV positive }\end{array}$ & \multirow{4}{*}{$\begin{array}{l}\text { Prophylaxis with clotting factors vs. } \\
\text { on-demand management }\end{array}$} & \multirow{4}{*}{$\begin{array}{l}\text { Germany, the } \\
\text { Netherlands, } \\
\text { Sweden, United } \\
\text { Kingdom, 2002 } \\
\text { (euro) }\end{array}$} & 83,100 & 0.059 & $\begin{array}{c}1,408,474 \\
(1,750,000)\end{array}$ \\
\hline & & $\begin{array}{l}\text { >30 years, } \\
\text { HIV positive }\end{array}$ & & & 96,337 & -0.030 & Dominated \\
\hline & & $\begin{array}{l}\leq 30 \text { years, } \\
\text { HIV negative }\end{array}$ & & & 83,100 & 0.033 & $\begin{array}{l}2,518,181 \\
(3,130,000)\end{array}$ \\
\hline & & $\begin{array}{l}\text { >30 years, } \\
\text { HIV negative }\end{array}$ & & & 96,337 & 0.019 & $\begin{array}{l}5,070,368 \\
(6,304,000)\end{array}$ \\
\hline \multirow{3}{*}{$\begin{array}{l}\text { Pattanaprateep } \\
\text { et al. }(2014)^{54}\end{array}$} & $\begin{array}{l}\text { Mild } \\
\text { hemophilia A } \\
\text { and B (with } \\
\text { no inhibitor } \\
\text { or }<5 \text { BU) }\end{array}$ & \multirow{3}{*}{ At birth } & \multirow{3}{*}{$\begin{array}{l}\text { Home-based care: FVIII and FIX } \\
\text { concentrate for treating early bleed- } \\
\text { ing episodes vs. treatment with } \\
\text { blood components when admitted }\end{array}$} & \multirow{3}{*}{$\begin{array}{l}\text { Thailand, } 2012 \\
\text { (Thai baht) }\end{array}$} & 227,377 & 2.820 & $\begin{array}{l}80,542 \\
(2,600)\end{array}$ \\
\hline & $\begin{array}{l}\text { Moderate } \\
\text { hemophilia A } \\
\text { and B (with } \\
\text { no inhibitor } \\
\text { or }<5 \text { BU) }\end{array}$ & & & & $-2,460,872$ & 5.970 & Dominant \\
\hline & $\begin{array}{l}\text { Severe } \\
\text { hemophilia A } \\
\text { and B (with } \\
\text { no inhibitor } \\
\text { or }<5 \text { BU) } \\
\end{array}$ & & & & $-5,718,732$ & 8.400 & Dominant \\
\hline
\end{tabular}

aICER stated in the study. The reported ICER is different from the ICER obtained by dividing incremental costs and QALYs stated in the study.

bBonn (high-dose) ITI protocol with $300 \mathrm{IU}$ per kg per day of rFVIII to eradicate inhibitors plus use of FVIII to control bleeds.

cLow-dose ITI protocol with $50 \mathrm{IU}$ per $\mathrm{kg}$ per day of $r$ FVIII to eradicate inhibitors plus use of FVIII to control bleeds.

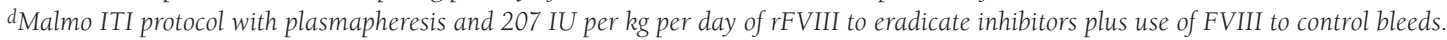

eTailored prophylaxis (escalating dose) beginning at a low frequency (FVIII 50 IU per kg once weekly) and escalating with repeated bleeding (maximum of FVIII 30 IU per kg twice weekly).

fLow-dose ITI protocol with 25 IU per kg per day of $r$ FVIII to eradicate inhibitors plus use of FVIII to control bleeds.

sSecondary prophylaxis with FVIII concentrates (on-demand treatment up to 2 years followed by prophylaxis).

hHybrid regimen with FVIII concentrates (initially receive prophylaxis until 18 years of age and then switch to treatment on-demand).

${ }^{i}$ Cost estimates are averaged across 4 countries stated in the study.

$A P C C=$ activated prothrombin complex concentrate; BU = Bethesda unit; FVIII=factor VIII; FIX=factor IX; HIV=human immunodeficiency virus; ICER=incremental cost-effectiveness ratio; ITI = immune tolerance induction; $\mathrm{FFVIII=} \mathrm{porcine} \mathrm{factor} \mathrm{VIII;} \mathrm{QALY=quality-adjusted} \mathrm{life-year;} r$ FVIIa= recombinant activated factor VII; $r$ FVIII = recombinant factor VIII; USD = U.S. dollars .

We identified 29 ICERs across 11 CUAs, which pertained to different country settings, treatment approaches, and patient populations. The median ICER for prophylactic treatment compared with on-demand treatment was $\$ 86,000$ per QALY for severe hemophilia A (range, dominant [i.e., more effective and less costly than the included comparator] to $\$ 869,000$ per QALY), $\$ 17,000$ per QALY for hemophilia B, and $\$ 46,000$ per QALY for studies that included patients with hemophilia A and B (range, dominant to $\$ 6,304,000$ per QALY). Three studies evaluated the cost-effectiveness of ITI for patients with hemophilia A with inhibitors compared with ondemand therapy with recombinant-activated FVIIa (rFVIIa); Knight et al. (2003) reported a median ICER of $\$ 169,000$ per QALY (range, dominant to $\$ 355,000$ per QALY), ${ }^{52}$ and Rasekh et al. (2011) and Earnshaw et al. (2015) reported that ITI was a dominant strategy compared with on-demand 
treatment. $^{53,55}$ Of the treatment regimens reviewed, treating hemophilia patients with inhibitors was reported to be the most cost-effective. The CUAs evaluated 3 ITI regimens for inhibitor eradication-Bonn protocol, low-dose protocol, and Malmo protocol. It was reported that all ITI protocols were dominant when compared with on-demand regimen. Among the limited number of studies that evaluated different ITI protocols compared to on-demand, the Malmo ITI protocol (extracorporeal immunoadsorption with protein A columns to remove high-titer inhibitory antibodies and rFVIII to eradicate inhibitors plus FVIII to control bleeds) was reported to be dominant (cost-saving) between Bonn ITI and low-dose ITI. Four studies evaluated the cost-effectiveness of prophylaxis treatment in patients with hemophilia with inhibitors, and the median ICER was $\$ 23,000$ per QALY (range, dominant to $\$ 355,000$ per QALY). Studies sponsored by pharmaceutical industries (CUAs, $\mathrm{n}=4$ ) reported a median ICER of $\$ 72,000$ per QALY for severe hemophilia A.

\section{Sensitivity Analyses}

Only 4 of 10 (40\%) studies performed probabilistic sensitivity analyses. In 1-way sensitivity analyses, the majority of studies reported the ICERs to be influenced by the cost of the clotting factors and the use/number of doses followed by discount rate. Other factors influencing the ICER were dosage of clotting factors, number of bleeds, and QOL values.

\section{Discussion}

The majority of reviewed studies were set outside the United States (82\%), most often in the United Kingdom (55\%) and Sweden (18\%), and more than half of the studies were published from 2009 through 2015. Hemophilia A was the most studied disease type (73\%). The majority of studies (64\%) were funded by the studied product's manufacturer. Treating hemophilia B was found to be more cost-effective compared with hemophilia A. Treatments for severe hemophilia A were associated with the highest cost-effectiveness ratios, with a median ICER of $\$ 86,000$ per QALY gained. Of patients with hemophilia A, those with high-titer inhibitors were those for whom treatment was estimated to be most cost-effective. Furthermore, patients treated with ITI were estimated to survive longer, with a reduction in the number of bleeding episodes and a corresponding increase in QALYs. The lifetime costs of patients treated with ITI were also estimated to be lower than those treated with on-demand therapies, leading to a more favorable cost-effectiveness ratio.

\section{Variation in Reported ICERs}

We found that the included CUAs reported a wide range of cost-utility ratios, from dominant to more than $\$ 6$ million per QALY. The median ICERs reported across the studies varied by hemophilia type: $\$ 86,000$ per QALY for studies evaluating severe hemophilia A treatment; $\$ 17,000$ per QALY for the study that evaluated hemophilia B treatments; and $\$ 46,000$ per QALY for studies including both patients with hemophilia A and B. Studies evaluating the cost-effectiveness of ITI for patients with inhibitors reported a wide range of ICERs compared with on-demand treatment (median ICERs in studies ranged from dominant to $\$ 169,000$ per QALY). CUAs examined a wide range of interventions-comparing different patterns of on-demand and prophylaxis treatments; tailored dosing of rFVIII; and dose modification of rFVIII in ITI to eradicate inhibitors and control bleeds. Also, 3 of 11 CUAs did not provide separate ICERs for patients with hemophilia A and hemophilia B. We note that the majority of industry-funded studies evaluated the cost-effectiveness of different dosing patterns and frequency of FVIII administration compared with on-demand therapy. The lack of consistency in the reported interventions makes it difficult to generalize the ICERs.

\section{Factors Explaining Variation in the Reported ICERs}

Study Perspective, Discounting, Time Horizon, and Study Sponsorship. We found that the majority of CUAs were conducted from a health care perspective and that the included studies applied different discount rates and time horizons. The application of different discount rates has the potential to impact cost-effectiveness estimates, particularly when the intervention has a preventive effect, as seen for prophylaxis regimens. The time horizons reported in the CUAs varied from 18 months to lifetime; the majority of CUAs reported a lifetime horizon. Sixty percent of the CUAs included in the sample were sponsored by product manufacturers. Evidence suggests that studies supported by industry tend to report more favorable ICERs than those not supported by industry. ${ }^{36}$ However, due to the small number of included studies, we were unable to compare the ICERs reported in industry- and nonindustryfunded studies.

Costs of Clotting Factors. Clotting factors have been estimated to account for approximately $90 \%$ of the direct health care costs for hemophilia management. ${ }^{37,38}$ We found that clotting factor cost varied in the identified studies, in part due to the different countries in which the studies were set. ${ }^{30}$ Also, disease severity dictates the amount and duration of clotting factors required for treatment; for instance, patients with severe hemophilia A require lifelong routine treatment, resulting in higher costs. ${ }^{39}$ Differences in unit costs and dosing regimens included in this analysis may also have affected the estimated ICERs. For example, Farrugia et al. (2013) reported the cost of FVIII (per $\mu$ g) as £0.61 in the United Kingdom, $\$ 0.95$ in the United States, and Swedish krona (SEK) 5.88 in Sweden, ${ }^{21}$ whereas Earnshaw et al. (2015) reported the cost of FVIII (per $\mu \mathrm{g}$ ) as $\$ 1.53 .{ }^{55} \mathrm{In}$ another example, the cost of orthopedic surgery varied across different countries ( $£ 745,065$ in the United Kingdom, \$1,245,995 in the United States, and SEK 8,372,742 in Sweden), while in another study, the cost of arthropathy surgery was $\$ 41,800 .^{21,55}$ 
Utility Weight Estimation. QALYs account for both length and quality of life. Weighting survival with a utility score for the particular health state incorporates QOL into the QALY calculation. There are various approaches to estimating utility weights-including direct elicitation methods, such as standard gamble, as well as time trade-off and indirect measurement methods, such as EuroQoL 5-Dimension and Short Form-36. Research has found that these methods can provide different results. For instance, research has found that the standard gamble approach often results in higher utility values compared with other approaches. ${ }^{40}$ However, other evidence suggests that incorporating alternative utility values into CEAs does not substantially alter study findings. ${ }^{41,42}$ In the present review, we found that the approaches used to elicit utility weights were poorly reported in the reviewed CUAs. Authors did not clearly state the measurement scale or the elicitation method used for each utility weight reported. Also, there was no information available on the sample population used.

\section{Recommendations for Future Research}

As a result of variations among the CUAs studied here, a consensus could not be reached on the cost-effectiveness of hemophilia treatments. Various guidelines for performing and reporting cost-effectiveness studies are currently available, and the recently released update on the U.S. panel of cost-effectiveness may reduce this variation and lead to greater standardization in the field. ${ }^{43}$

Further research on the cost-effectiveness of hemophilia treatments is needed. We found that treatments for hemophilia B were less well studied than treatments for hemophilia A and that many studies did not differentiate between hemophilia types when reporting cost-effectiveness.

The CUAs included in this review generally accounted for joint bleeds and their consequences, likely because they are the most common and easily quantifiable complications of hemophilia. Future studies should also account for other rare but costly complications, such as bleeding into the central nervous system, the gastrointestinal tract, and the renal system. ${ }^{44}$ Accounting for these clinical complications in the model or clinical trial will provide a more accurate estimate of cost-effectiveness.

\section{Limitations}

Our study has a number of important limitations. By limiting our systematic review to CUAs, we did not include studies reporting cost-effectiveness in terms of clinical endpoints (e.g., the cost per bleeding episode avoided) or studies reporting cost-effectiveness in terms of the cost per life year gained.

Because a number of studies combined patients with hemophilia A and B in the study patient population, our findings were limited. Generalizing study findings to both patient populations may be misleading, as the prevalence and costs associated with hemophilia A and B treatment vary considerably, with hemophilia A estimated to be associated with higher annual costs. ${ }^{45}$

The number of identified studies was insufficient to allow us to stratify the dataset. A larger sample would have allowed for a comparison of ICERs for patients with and without inhibitors, for patients suffering from comorbidities and infections with those who were not, and by hemophilia type. Furthermore, there was lack of consistency across the reported interventions (from daily prophylaxis to different ITI regimens), making it difficult to generalize the ICERs. Most of the CUAs were based on populations outside of the United States, which made it difficult to understand the implication on U.S. health care costs.

This study does not include the effect of treatment on productivity of patients (work or school absenteeism, presenteeism, income loss) and caregivers (unpaid hemophilia-related support) or other spillover costs in sectors like education or consumption costs from patients living longer. The Second Panel on Cost-Effectiveness in Health and Medicine recommends incorporating a societal perspective while conducting cost-effectiveness analyses. ${ }^{43}$

This systematic review did not include CUAs for the recently approved FDA drugs (long-acting factors).

\section{Comparison of Study Findings to Systematic Reviews of CEAs of Orphan Drugs or Rare Diseases}

We attempted to compare our findings with reviews of the cost-effectiveness of other rare diseases-diseases defined as having a prevalence of 5 or less out of 10,000 individuals in the European Union and diseases that affect fewer than 200,000 individuals in the United States $2,3,46$ —but only identified a single study that reviewed the cost-effectiveness of orphan drugs in cancer. In a systematic review of oncology orphan drugs, Cheng et al. (2012) identified 21 cost-effectiveness studies for 47 oncology orphan drugs. ${ }^{47}$ Included among these cost-effectiveness studies were 14 CUAs, which reported ICERs up to roughly $\$ 240,000$ per QALY. ${ }^{47}$ Our findings suggest that the cost-effectiveness of hemophilia treatments compare favorably to treatments for other orphan diseases.

\section{Considering the Value of Orphan Drugs}

Because of the rarity of disease and high treatment costs, it has been suggested that a different cost-effectiveness threshold should apply to orphan drugs. However, a consensus on how this value should be calculated is unclear. ${ }^{48}$ Another suggested approach is to apply "equity weights" based on disease prevalence to health outcomes in cost-effectiveness analyses. ${ }^{49}$ Guidance issued by the National Institute of Health and Care Excellence (NICE) in the United Kingdom suggests that weighting outcomes, for example, for end-of-life care, is an approach that allows for societal preferences to be accounted for in costeffectiveness analyses. However, this approach has not yet been applied to orphan drugs. ${ }^{50}$ 
A greater understanding of how to value hemophilia treatments may be important when assessing the value of future novel therapies (e.g., gene therapy). Recent experience with hepatitis $C$ treatments has shown that despite being curative for some patients, their high cost can make them unaffordable for many. Ultimately, health care systems will need to devise new mechanisms to control costs and provide access to highly effective therapies.

\section{Conclusions}

The cost-effectiveness of hemophilia treatments varied based on the treatment approach, patient characteristics, and disease severity. Identified estimates of the cost-effectiveness of hemophilia treatments are broadly comparable to those of other orphan drugs.

\section{Authors}

TEJA THORAT, MSc, MPH; PETER J. NEUMANN, ScD; and JAMES D. CHAMBERS, PhD, MPharm, MSc, Center for the Evaluation of Value and Risk in Health, Institute for Clinical Research and Health Policy Studies, Tufts Medical Center, Boston, Massachusetts.

AUTHOR CORRESPONDENCE: Teja Thorat, MSc, MPH, Manager, Health Economics and Outcomes Research, Vertex Pharmaceuticals, 50 Northern Ave., Boston, MA 02210. Tel.: 617.961.7187;

E-mail: teja_thorat@vrtx.com.
5. Roberts HR, Key NS, Escobar MA. Hemophilia A and hemophilia B. In: Kaushansky K, Lichtman M, Beutler E, et al, eds. Williams Hematology. 8th ed. New York: McGraw Hill; 2010:2009-30.

6. World Federation of Hemophilia. Report on the Annual Global Survey 2016. October 2017. Available at: http://wwwl.wfh.org/publication/files/pdf1690.pdf. Accessed Jun 9, 2018.

7. Bolton-Maggs PH, Pasi KJ. Haemophilias A and B. Lancet. 2003;361(9371): 1801-09.

8. White GC, Rosendaal F, Aledort LM, Lusher JM, Rothschild C, Ingerslev J Definitions in hemophilia. Recommendation of the scientific subcommittee on factor VIII and factor IX of the scientific and standardization committee of the International Society on Thrombosis and Haemostasis. Thromb Haemost. 2001;85(3):560

9. Srivastava A, Brewer AK, Mauser-Bunschoten EP, et al. Guidelines for the management of hemophilia. Haemophilia. 2013;19(1):el-e47.

10. Mannucci PM, Tuddenham EG. The hemophilias-from royal genes to gene therapy. N Engl J Med. 2001;344(23):1773-79.

11. Gringeri A, Ewenstein B, Reininger A. The burden of bleeding in haemophilia: is one bleed too many? Haemophilia. 2014;20(4):459-63.

12. Valentino LA. Blood-induced joint disease: the pathophysiology of hemophilic arthropathy. J Thromb Haemost. 2010;8(9):1895-902.

13. Van Den Berg HM, Dunn A, Fischer K, Blanchette VS. Prevention and treatment of musculoskeletal disease in the haemophilia population: role of prophylaxis and synovectomy. Haemophilia. 2006;12 Suppl 3:159-68.

14. Young G. New challenges in hemophilia: long-term outcomes and complications. Hematology Am Soc Hematol Educ Program. 2012;2012:362-68.

15. Dekoven M, Wisniewski T, Petrilla A, et al. Health-related quality of life in haemophilia patients with inhibitors and their caregivers. Haemophilia. 2013;19(2):287-93.

16. Gringeri A, Mantovani LG, Scalone L, Mannucci PM. Cost of care and quality of life for patients with hemophilia complicated by inhibitors: the COCIS Study Group. Blood. 2003;102(7):2358-63.

17. Manco-Johnson MJ, Abshire TC, Shapiro AD, et al. Prophylaxis versus episodic treatment to prevent joint disease in boys with severe hemophilia N Engl J Med. 2007;357(6):535-44.

18. Ingerslev J, Lethagen S, Hvitfeldt PL, et al. Long-standing prophylactic therapy vs. episodic treatment in young people with severe haemophilia: a comparison of age-matched Danish and Russian patients. Haemophilia. 2014;20(1):58-64

19. Royal S, Schramm W, Berntorp E, et al. Quality-of-life differences between prophylactic and on-demand factor replacement therapy in European haemophilia patients. Haemophilia. 2002;8(1):44-50

20. Colombo GL, Di Matteo S, Mancuso ME, Santagostino E. Cost-utility analysis of prophylaxis versus treatment on demand in severe hemophilia A. Clinicoecon Outcomes Res. 2011;3:55-61.

21. Farrugia A, Cassar J, Kimber MC, et al. Treatment for life for severe haemophilia A-a cost-utility model for prophylaxis vs. on-demand treatment. Haemophilia. 2013;19(4):e228-38

22. Zhou ZY, Koerper MA, Johnson KA, et al. Burden of illness: direct and indirect costs among persons with hemophilia A in the United States. J Med Econ. 2015;18(6):457-65.

23. Eldar-Lissai A, Hou Q, Buckley BC, Zhou J, Krishnan S. The changing costs of caring for hemophilia patients in the US. Poster presented at: Academy of Managed Care 2015 Annual Meeting \& Expo; April 7-10, 2015; San Diego, CA.

24. National Hemophilia Foundation. MASAC recommendation concerning prophylaxis (regular administration of clotting factor concentrate to prevent bleeding). MASAC Document \#179. February 28, 2016. Available at: http://www.hemophilia.org/NHFWeb/Resource/StaticPages/menu0/menu5/ menu57/masac179.pdf. Accessed May 23, 2018 
25. Globe DR, Curtis RG, Koerper MA. Utilization of care in haemophilia: a resource-based method for cost analysis from the Haemophilia Utilization Group Study (HUGS). Haemophilia. 2004;10 Suppl 1:63-70.

26. Goudemand J. Hemophilia. Treatment of patients with inhibitors: cost issues. Haemophilia. 1999;5(6):397-401.

27. DiMichele DM. Immune tolerance induction in haemophilia: evidence and the way forward. J Thromb Haemost. 2011;9(Suppl 1):216-25.

28. Athale AH, Marcucci M, Iorio A. Immune tolerance induction for treating inhibitors in people with congenital haemophilia A or B. Cochrane Database Syst Rev. 2014;4:CD010561.

29. Colowick AB, Bohn RL, Avorn J, Ewenstein BM. Immune tolerance induction in hemophilia patients with inhibitors: costly can be cheaper. Blood. 2000;96(5):1698-702

30. Carcao M, Ungar WJ, Feldman BM. Cost-utility analysis in evaluating prophylaxis in haemophilia. Haemophilia. 2004;10 Suppl 1:50-57.

31. Drummond MJ, Sculpher MJ, Torrance GW, O'Briend BJ, Stoddart GL. Methods for the Economic Evaluation of Health Care Programmes. New York: Oxford University Press; 2005.

32. Lippert B, Berger K, Berntorp E, et al. Cost effectiveness of haemophilia treatment: a cross-national assessment. Blood Coagul Fibrinolysis. 2005;16(7):477-85

33. Risebrough N, Oh P, Blanchette V, Curtin J, Hitzler J, Feldman BM. Cost-utility analysis of Canadian tailored prophylaxis, primary prophylaxis and on-demand therapy in young children with severe haemophilia A. Haemophilia. 2008;14(4):743-52

34. Neumann PJ, Greenberg D, Olchanski NV, Stone PW, Rosen AB. Growth and quality of the cost-utility literature, 1976-2001. Value Health. 2005;8(1):3-9.

35. Thorat T, Cangelosi M, Neumann PJ. Skills of the trade: the Tufts Cost-Effectiveness Analysis (CEA) Registry. J Ben-Cost Anal. 2012;3:1-7.

36. Bell CM, Urbach DR, Ray JG, et al. Bias in published cost effectiveness studies: systematic review. BMJ. 2006;332(7543):699-703.

37. Bohn RL, Avorn J, Glynn RJ, Choodnovskiy I, Haschemeyer R, Aledort LM. Prophylactic use of factor VIII: an economic evaluation. Thromb Haemost. 1998;79(5):932-37.

38. Miners AH, Sabin CA, Tolley KH, Lee CA. Cost-utility analysis of primary prophylaxis versus treatment on-demand for individuals with severe haemophilia. Pharmacoeconomics. 2002;20(11):759-74.

39. Miners A. Revisiting the cost-effectiveness of primary prophylaxis with clotting factor for the treatment of severe haemophilia A. Haemophilia. 2009;15(4):881-87.

40. Dolan P, Sutton M. Mapping visual analogue scale health state valuations onto standard gamble and time trade-off values. Soc Sci Med. 1997;44(10):1519-30
41. Chapman RH, Berger M, Weinstein MC, Weeks JC, Goldie S, Neumann PJ. When does quality-adjusting life-years matter in costeffectiveness analysis? Health Econ. 2004;13(5):429-36.

42. Greenberg D, Neumann PJ. Does adjusting for health-related quality of life matter in economic evaluations of cancer-related interventions? Expert Rev Pharmacoecon Outcomes Res. 2011;11(1):113-19.

43. Neumann PJ, Sanders GD, Russell LB, Siegel JE, Ganiats TG Cost-Effectiveness in Health and Medicine. 2nd ed. New York: Oxford University Press; 2016.

44. Farrugia A, O'Mahony B, Cassar J. Health technology assessment and haemophilia. Haemophilia. 2012;18(2):152-57.

45. Saxena K. Barriers and perceived limitations to early treatment of hemophilia. J Blood Med. 2013;4:49-56.

46. Simoens S. Pricing and reimbursement of orphan drugs: the need for more transparency. Orphanet J Rare Dis. 2011;6:42.

47. Cheng MM, Ramsey SD, Devine EB, Garrison LP, Bresnahan BW, Veenstra DL. Systematic review of comparative effectiveness data for oncology orphan drugs. Am J Manag Care. 2012;18(1):47-62.

48. Hughes DA, Tunnage B, Yeo ST. Drugs for exceptionally rare diseases: do they deserve special status for funding? 2JM. 2005;98(11):829-36.

49. Drummond MF, Wilson DA, Kanavos P, Ubel P, Rovira J. Assessing the economic challenges posed by orphan drugs. Int J Technol Assess Health Care. 2007;23(1):36-42

50. National Institute for Health and Clinical Excellence. Appraising lifeextending, end of life treatments. Available at: https://www.nice.org.uk/guidance/gid-tag387/documents/appraising-life-extending-end-of-life-treatmentspaper2. Accessed May 23, 2018.

51. Ekert H, Brewin T, Boey W, Davey P, Tilden D. Cost-utility analysis of recombinant factor VIIa (NovoSeven) in six children with long-standing inhibitors to factor VIII or IX. Haemophilia. 2001;7(3):279-85.

52. Knight C, Paisley S, Wight J, Jones ML. Economic modelling of different treatment strategies for haemophilia A with high-responding inhibitors. Haemophilia. 2003;9(4):521-40

53. Rasekh HR, Imani A, Karimi M, Golestani M. Cost-utility analysis of immune tolerance induction therapy versus on-demand treatment with recombinant factor VII for hemophilia A with high titer inhibitors in Iran. Clinicoecon Outcomes Res. 2011:3:207-12.

54. Pattanaprateep O, Chuansumrit A, Kongsakon R. Cost-utility analysis of home-based care for treatment of Thai hemophilia A and B. Value Health Reg Issues. 2014;3C:73-78

55. Earnshaw SR, Graham CL, McDade JB, Spears, Kessler CM. Factor VIII alloantibody inhibitors: cost analysis of immune tolerance induction vs. prophylaxis and on-demand with bypass treatment. Hemophilia. 2015;21:310-19. 